\title{
Genetic inference of group dynamics and female kin structure in a western lowland gorilla population (Gorilla gorilla gorilla)
}

\author{
M. Arandjelovic, J. Head, C. Boesch, M. M. Robbins, and L. Vigilant \\ Max Planck Institute for Evolutionary Anthropology, Deutscher Platz 6, 04103 Leipzig, Germany \\ Correspondence to: M. Arandjelovic (arandjel@eva.mpg.de)
}

Received: 15 September 2014 - Revised: 20 November 2014 - Accepted: 8 December 2014 - Published: 15 December 2014

\begin{abstract}
Dispersal and grouping patterns form the foundations of social interactions in group-living mammals and are the outcomes of a complex interplay between inbreeding avoidance, kin cooperation and competition, predation pressure and food resource distribution. In species where both sexes disperse, the potential for kinbiased associations would seem limited. In one such species, the western lowland gorilla (WLG), short-term data suggest that female kin associations may be present due to directed local dispersal decisions, but monitoring of groups over longer timescales is needed to better elucidate this pattern. Using autosomal genotyping of 419 faecal samples representing 85 unhabituated gorillas collected non-invasively over 5 years in a $132 \mathrm{~km}^{2}$ section of Loango National Park, Gabon, we investigated the dynamics of WLG group composition, social structure and patterns of dispersal. By revealing two group dissolutions, one group formation and the movement of 13 gorillas between groups, this study demonstrates the utility of genetic analysis as a way to track individuals, groups and population dynamics on a larger scale than when monitoring the behaviour of a limited number of habituated groups or through one-time genetic sampling. Furthermore, we find that females are found in groups containing their female kin more often than expected by chance, suggesting that dispersal may not impede female kin associations in WLGs.
\end{abstract}

\section{Introduction}

Group living in many mammal species is generally explained as an arrangement that maximizes the potential benefits of kin cooperation and predator avoidance (Clutton-Brock and Lukas, 2012; Handley and Perrin, 2007). The potential costs of inbreeding and competition, particularly with kin, are usually invoked to explain the prevalence of sex-biased natal dispersal in mammals (Handley and Perrin, 2007; Thierry, 2008). However, the dispersing sex would seem to lose the potential for fitness-enhancing interactions with life-long associates who may also be close kin (Langergraber et al., 2007, 2009; Silk, 2009; Silk et al., 2006). Unlike most mammal species which display predominantly male dispersal (Greenwood, 1980), great apes such as eastern gorillas, western lowland gorillas (WLGs, Gorilla gorilla gorilla), bonobos and chimpanzees typically exhibit dispersal of females from the natal group at maturity (Greenwood, 1980;
Pusey and Packer, 1987; Robbins, 2010; Wrangham, 1979), and these females may disperse to other social units multiple times in their lives (Boesch, 2009; Stokes et al., 2003). WLG males also consistently disperse from their natal group and become solitary or reside in non-reproductive social units before possibly acquiring females and forming their own reproductive groups. These reproductive groups nearly always contain only a single fully mature adult (silverback) male along with mature females and immatures of both sexes (Bradley et al., 2004; Gatti et al., 2004; Robbins et al., 2004). However, unlike most harem species, including mountain gorillas (Watts, 2000), hamadryas baboons (Swedell and Tesfaye, 2003), plains zebras (Rubenstein, 1986) and greater sac-winged bats (Voigt and Streich, 2003), neither internal ("queuing") nor external group takeovers by adult silverbacks have been observed in WLGs (Harcourt and Stewart, 2007). Instead, new groups may form when females join lone silverback males or females transfer to existing groups dur- 
ing intergroup encounters. It has been suggested that WLGs, with routine dispersal by both sexes, may be one of the best models for studying early hominid evolution (Koenig and Borries, 2012).

It is currently thought that female gorillas gain little from residing with kin but obtain benefits from dispersing by avoiding potential inbreeding in their natal community and by having the ability to choose a new male in a new group (Breuer et al., 2012; Caillaud et al., 2008; Guschanski et al., 2008; Harcourt and Stewart, 2007). However, genetic analysis of multiple adjacent WLG groups at one site suggested that, despite routine dispersal by both sexes, female WLGs may maintain kin associations post-emigration (Bradley et al., 2007). Furthermore, in mountain gorillas, a WLG sister taxon displaying non-obligate female and male dispersal, non-dispersing related females are more tolerant of each other than of non-relatives (Watts, 1994a, b). On the other hand, Douadi et al. (2007) suggested that the proportion of related WLG female dyads (including both adults and immatures) within groups did not differ from the proportion between groups, while another study found few WLG female kin dyads in their study population (Inoue et al., 2013). However, the latter two studies relied upon estimation of dyadic relatedness values, which is not considered an accurate way to identify related pairs in a population (Csillery et al., 2006), thus leaving the question of female kin associations in WLGs unresolved.

Because female gorillas only transfer between groups during intergroup encounters or after group dissolution and are never solitary (Harcourt and Stewart, 2007), the timing and distance of single dispersal events are limited. Furthermore, because the risk of infanticide would appear to inhibit transfer by females with dependent young, opportunities for female dispersal are constrained in comparison to males (Robbins et al., 2013; Robbins, 2010). Nevertheless, females may move across multiple groups over a lifetime as the result of multiple transfers which are believed to reflect female choice (Breuer, 2010; Jeffery et al., 2007; Robbins et al., 2004). While females typically disperse singly, the transfer of pairs of females has been inferred twice, with two females observed in one group, and shortly thereafter in a new group after the death of their group's silverback (Parnell, 2002; Stokes et al., 2003). Such transfers subsequent to group dissolution are generally considered involuntary in nature. Based on these considerations and the observation of higher than expected average relatedness among females within groups at one site, it is hypothesized in WLGs that females may be able to maintain kin relationships despite natal and secondary dispersal (Bradley et al., 2007), which could in turn confer fitness benefits (Chapais, 2001; Clutton-Brock and Huchard, 2013; Hatchwell, 2010).

The dynamics of WLG group composition have been observed at several study sites (Bermejo, 2004; Cipolletta, 2004; Doran-Sheehy et al., 2004; Gatti et al., 2004; Jeffery et al., 2007; Magliocca et al., 1999; Parnell, 2002; Robbins et al., 2004; Stokes et al., 2003; Tutin, 1996), and these studies have elucidated some of the apparent contrasts between WLGs and mountain gorillas. For example, all-male bachelor groups, mixed-sex non-reproductive groups and single-male breeding groups have all been documented in WLGs, but in contrast to mountain gorillas, multi-male breeding groups have not been observed except for a few transient cases (Gatti et al., 2004; Jeffery et al., 2007; Magliocca et al., 1999; Parnell, 2002; Robbins et al., 2004; Stokes et al., 2003; Tutin, 1996). Furthermore, group fissions (the splitting of one group into two or more) have also not been observed in WLGs, and the only mode of group formation observed has been by the acquisition of females by lone silverback males (Gatti et al., 2004; Robbins et al., 2004). Group dissolutions (the cessation of existence of a group, wherein the group silverback no longer retains any females and females transfer to several different new groups) have been observed at one WLG site at a rate approximately 5 times higher than in mountain gorillas (Robbins et al., 2004). In general, however, the difficulty of simultaneous observational monitoring of multiple groups of a long-lived species has limited the direct study of patterns of dispersal and group formation in WLGs.

Genetic analyses provide an opportunity for understanding the dispersal choices of individuals in such elusive species. In this study we use samples collected within a $132 \mathrm{~km}^{2}$ area over a total of 33 months spanning 5 years in Loango National Park, Gabon, and employ autosomal microsatellite genotyping in conjunction with parentage analysis to determine the composition of groups in the study area. We take advantage of the temporal depth of the sampling to focus on group dynamics and to estimate the frequency of group formations, dissolutions and individual dispersal events. Finally, we use relatedness analyses to test the hypotheses that female WLGs are found in proximity to their same-sex kin post-dispersal.

\section{Methods}

\subsection{Study site and sample collection}

As detailed previously, we opportunistically collected a total of 396 gorilla faecal samples across a $132 \mathrm{~km}^{2}$ area in the central sector of Loango National Park, Gabon (Head et al., 2011), between February 2005 and September 2007 (Arandjelovic et al., 2010). We collected an additional 23 samples from the same area from March to April 2009. We preserved the faeces using the two-step ethanol-silica procedure (Nsubuga et al., 2004). The gorillas in the research area largely make their night nests in trees, making age-class designation based on faecal bolus size unfeasible as the fallen faeces break up upon impact with the forest floor (Robbins et al., 2004; Schaller, 1963). Over the course of the sampling, some of the gorilla groups were being followed for habituation to human presence, and although they were never fully habituated over the course of this study, the number of groups 
present at Loango and the composition of several groups were inferred by direct observation and through a parallel camera trap study (Head et al., 2011, 2013). Observations on the presence and composition of groups, along with a GPS waypoint recording, were made opportunistically by the habituation teams when groups were encountered. Groups were observed from a few minutes to up to $6 \mathrm{~h}$ during the course of sample collection for this study. The identity of the group can often be inferred by visually confirming the identity of the silverback of the group by distinct markings and characteristics on the face or body. The named groups (Tonda, Mandondo, Achilles, LayonA group and Indegho) were all known to be unique groups from direct observations, although their exact group composition was not fully confirmed. Samples were collected from beneath night nests and from where gorillas had defecated as they moved through the forest during the day. We recorded the geographic coordinates of each faecal sample using a Garmin GPSMap ${ }^{\circledR} 60$ or 60CSx. As chimpanzees and gorillas live sympatrically at Loango, we used the STRUCTURE 2.1 Bayesian model-based clustering program (Pritchard et al., 2000), which reliably differentiates samples of gorilla origin from misidentified chimpanzee faecal remains based on the allele frequencies present in the two species (Arandjelovic et al., 2010). WLGs are listed as critically endangered by the IUCN (Walsh et al., 2008) and all samples were collected non-invasively without contact with any of the animals under study, and thus no ethical consent was required for the research. All research was carried out with permission from the Agence Nationale des Parcs Nationaux (ANPN) and the Centre National de la Recherche Scientifique et Technique (CENAREST) of Gabon.

\subsection{DNA extraction, quantification and amplification}

We stored samples for up to 1 year after collection and extracted DNA from them using the QIAmp Stool kit (QIAGEN) with slight modifications (Nsubuga et al., 2004). We determined the sex of the gorillas by amplifying a portion of the amelogenin locus (Bradley et al., 2000). We also amplified the samples at 16 autosomal microsatellite loci using a two-step multiplex PCR method described in detail elsewhere (Arandjelovic et al., 2009, 2010). We combined up to four different PCR products and electrophoresed them on an ABI PRISM 3100 genetic analyser. We sized alleles relative to an internal size standard (ROX-labelled HD400) using GeneMapper software version 3.7 (Applied Biosystems). For accuracy, we validated heterozygous genotypes by observing each allele in two or more independent reactions (Arandjelovic et al., 2009). To ensure with $>99 \%$ certainty that homozygote genotypes were authentic and not the result of allelic dropout, we analysed results from up to five successful independent PCR amplifications, depending on the amount of DNA in the sample (Arandjelovic et al., 2009), as determined by the DNA quantification method described in Morin et al. (2001).

\subsection{Discrimination of individuals and group membership}

We used CERVUS 3.0 to compare genotypes to detect cases of different samples originating from the same individual. For every case in which genotypes from two samples had no mismatches and were identical at a minimum of seven loci, the probability that two genotypes were exact matches despite originating from two different individuals $\left(P_{\mathrm{IDsibs}}\right)$ was $<0.001$, so we are confident that relatives were not misclassified as the same individual (Waits et al., 2001). We were able to genotype one sample (G86) at only four loci (with confirmed alleles at an additional four loci) and three samples at only six loci (G68, G97 and G98); however, these samples mismatched all other samples at a minimum of one locus.

We evaluated minimum group membership as detailed in Arandjelovic et al. (2010). In brief, we first assumed that when samples from two or more individuals were collected on the same day at the same GPS location (same nest site or multiple fresh faecal remains found within $20 \mathrm{~m}$ of each other), these samples belonged to the same group. Once individuals were assigned to a group, when samples from those individuals were then identified on other sampling days, even if they were collected alone, then those individuals were still considered part of the initial group assignment. Some identified groups may thus actually be part of a larger group that was not well sampled over time due to the heterogeneous and opportunistic sampling protocol used (Arandjelovic et al., 2010). However, for the purposes of these analyses, for lack of contrary evidence, and supported by the identification of at least eight breeding groups at Loango by remote camera traps (Head et al., 2013), we treat each identified group as its own social unit. When we detected an individual in a particular group and then later on in the study period in another group, we identified this as a dispersal event. We can only assume that we have minimum group membership, as some individuals may by chance not have been sampled and/or genotyped from any of the groups. Lone silverback males are defined as males that we sampled more than once and always found alone. We consider individuals that were sampled only once and alone to have an unknown social unit affiliation.

To calculate the rate of dispersal and rate of group dissolutions, we estimated the number of recorded gorilla social unit years (sum of years each social unit was observed) by taking the total number of months between the initial and final sampling of any individual in any given gorilla social unit (group or lone silverback). Because we did not sample all individuals consistently over the study period and large time gaps occur between records of the same group, this is a very approximate measure of total detection time. With these caveats in mind, we have a total of 19 gorilla social unit years recorded. 


\subsection{Partial pedigree reconstruction}

We reconstructed partial pedigree relationships for individuals in the Loango gorilla study population using parentage analysis to identify possible offspring in the data set. We first did a simulation of parentage analysis in CERVUS 3.0 to determine the power of our microsatellite loci to assign motherfather-offspring trios with 85,95 and $99 \%$ confidence in our data set. We simulated genotypes for 10000 offspring using the allele frequencies from our data set. We then ran a series of assignment simulations, assuming that we sampled from $30 \%$ of mothers and fathers. Analyses were repeated assuming 20 and $10 \%$ of parents were sampled to ensure results were robust (data not shown). The proportion of genotyped loci was $88 \%$ and we conservatively allowed for a $1 \%$ genotyping error rate.

In the parentage analysis, we used all males as potential fathers, all females as potential mothers and all individuals as potential offspring and allowed up to one mismatch for parent-offspring trios to account for genotyping errors and mutational events. Firstly, we assigned paternity and maternity when the trio-confidence score was $>85 \%$ for individuals genotyped at a minimum of 14 loci. The majority of parentage trios $(N=15 / 17)$ met this criterion. In $4 / 15$ of these cases, one mismatch occurred in the trio and could be attributed to either possible dropout in the offspring's genotype $(N=1)$, genotyping error because only one sample from the individual was genotyped $(N=2)$ or a mutation in the offspring's genotype $(N=1)$. In two additional instances the candidate sire and dam shared an allele at all loci and thus represented possible parent-offspring relationships. In these two cases, however, the individuals had been assigned to other trios and we accepted these trios, albeit with lower certainty $(<85 \%)$. In total we assigned 17 parent-offspring trios. We considered any group male with assigned offspring a group silverback. We also assigned a male identified as lone silverback (samples found alone multiple times; Arandjelovic et al., 2010) as a father and assumed that he was a deposed male that had sired offspring when previously leading a group. We consider individuals within groups that share an allele at all loci but that are not assigned in the trio analysis as probable first-order relatives, although whether they are parent-offspring, full siblings or half-siblings cannot be determined (Blouin et al., 1996; Csillery et al., 2006). We refer to groups as "breeding groups" if we identified at least one parent-offspring trio in the group.

\subsection{Female relatedness}

We evaluated dyadic relatedness $(R)$ using the Lynch and Ritland (1999) estimator implemented in the software KINGROUP (Konovalov et al., 2004) as it has been shown to minimize the sampling variance in most populations (Csillery et al., 2006). We repeated all analyses using the Queller and Goodnight relatedness estimator (Queller and
Goodnight, 1989) with consistent results (data not shown) unless otherwise noted. Pairwise relatedness estimates are associated with large variances due to random differences among loci in sharing of alleles that are identical by descent as well as in the chance sharing of alleles that are identical by state (Blouin, 2003; Queller and Goodnight, 1989). Therefore, use of a limited number of microsatellite loci for classification of dyads tends to result in a high misclassification rate (Csillery et al., 2006; Van Horn et al., 2008). However, relatively few loci are adequate for estimating $a v-$ erage relatedness within and across groups, as long as comparisons between sets of individuals are done using an approach such as permutation tests that take into account the non-independence of data points (Blouin, 2003; Lukas et al., 2005; Queller and Goodnight, 1989). For the following analyses, we removed from the data set all within-group individuals that shared an allele at all loci with the group silverback, as these are considered potential offspring (i.e. pre-dispersal immature individuals). Furthermore, we only included individuals present in the data set from June 2006 to September 2007 since observational and genetic information suggested that the groups were stable with no transfers during this time period (and therefore each individual was attributed to only a single group). We then performed three different permutation analyses to examine the distribution of female kin at Loango.

First, we tested whether females in the study area are more related than expected by chance. To do so, we estimated average $R$ for the 36 females present from 2006 to 2007. We then pooled all male and female samples $(N=59)$ together and calculated average relatedness of a random draw of 36 individuals 10000 times using a Microsoft Excel macro written by Lukas et al. (2005). We then compared average dyadic relatedness of all females in the population to the distribution of permuted relatedness values of males and females combined (Lukas et al., 2005).

Second, we tested whether females occur in groups containing kin more often than expected by chance (Bradley et al., 2007). In the Loango research area, like at all WLG research sites, there is a high degree of home range overlap for all of the study groups (Arandjelovic et al., 2010); thus even if females are limited to transferring to neighbouring groups, we assume in this test that all groups in the study area are equally possible transfer options. We calculated the average dyadic relatedness of females within groups by averaging across all pairwise comparisons and comparing this to the distribution of average relatedness values obtained by permutation analysis under a simplified model of random dispersal (Bradley et al., 2007; Lukas et al., 2005; Manly, 1997). For the permutation, we pooled all females found in groups from 2006 to $2007(N=35)$, resampled them into groups (holding number of groups $(N=8)$ and size of groups constant) 10000 times and then calculated average pairwise relatedness for these randomly constructed groupings using a Microsoft Excel macro written by Lukas et al. (2005). 


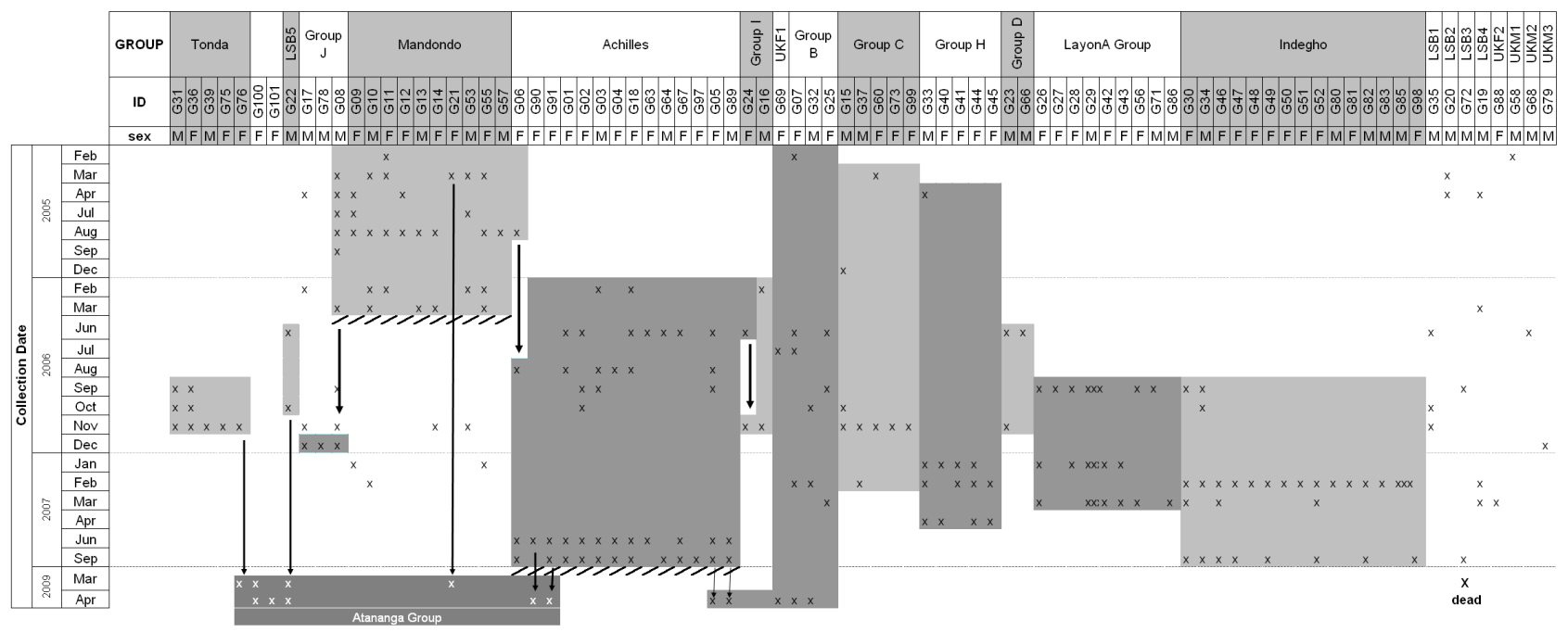

Figure 1. The inferred composition and dynamics of the Loango gorilla groups over the 5-year study period. Crosses designate that the gorilla was detected at least once in the corresponding month. Black arrows depict transfers between social units. Hatched lines represent the time at which the last nest group was found from the Mandondo or Achilles groups. LSB: lone silverback male; UKF: unknown female; UKM: unknown male. Unknown individuals were captured alone and once only and thus have no social unit affiliation. To calculate the number of recorded gorilla social unit years, we took the total number of months between the initial and final samplings of any individual in any given gorilla social unit (group or LSB) which corresponds to the shaded area for each group.

Third, because we cannot be sure that all groups are equally likely transfer options as assumed in the second analyses above, we tested whether females occur in groups containing kin more often than expected by a scenario where females preferentially disperse to neighbouring groups. To account for the possibility that dispersal to closer groups is more likely than dispersal to more distant groups, we permuted the females such that they had higher likelihoods of being redistributed to geographically closer groups. Specifically, we assigned groups with probabilities equaling $1 /(1+$ distance), with distance being the distances between the centre points of the groups' home ranges in kilometres. Females occurring in the same group were given probabilities equaling those for their nearest neighbour as to reduce the bias towards related individuals being assigned to the same group (i.e. females in the same group would otherwise have a group assignment probability of $1 /(1+0)=1)$. For the permutation, we again pooled all females found in groups from 2006 to 2007 ( $N=35$ ), resampled them into groups (holding number of groups $(N=8)$ and size of groups constant) 1000 times and then calculated average pairwise relatedness for these randomly constructed groupings in $\mathrm{R}$ as programmed by R Core Team (2013).

\section{Results}

\subsection{Group structure and individual movements}

Using genetic analysis, we identified a total of 85 different gorillas during $2005-2009$ in a $132 \mathrm{~km}^{2}$ area of Loango
National Park. We identified 18 social units made up of 12 groups and 6 lone silverback males (males that were sampled more than once and always alone) over the course of the study (Fig. 1 and Fig. S1 in the Supplement). Three additional males and one female could not be attributed to any social unit as they were each only collected once.

During the study, two of the groups apparently dissolved (Mandondo and Achilles) as we never resampled the individuals together nor were the groups directly observed after the suggested dissolution date (Figs. 1, 2a). Furthermore, when individuals from these groups were resampled they were either outside of their original range or found in association with new groups. Two groups formed over the course of the study (group J, a potential all-male group, and Atananga), both mostly comprising individuals previously found in association with other social units. Sample collection in 2009 was motivated by the suspected formation of a new group from direct observations. Even though only 23 samples were collected in 2009, these samples originated from five nest groups (allowing for repeated sampling, and therefore linking, of individuals found at the same site) and represent individuals from only two groups: Atananga (resampled from four nest group sites) and group B (sampled at one nest group site and containing three individuals detected from 2006 to 2007).

We confirmed the formation of the new Atananga group and deduced that its members consisted of a previously identified lone silverback male, four previously identified females from three different groups (two of which had dissolved) and two females of unknown origin. The two females that origi- 
(a)

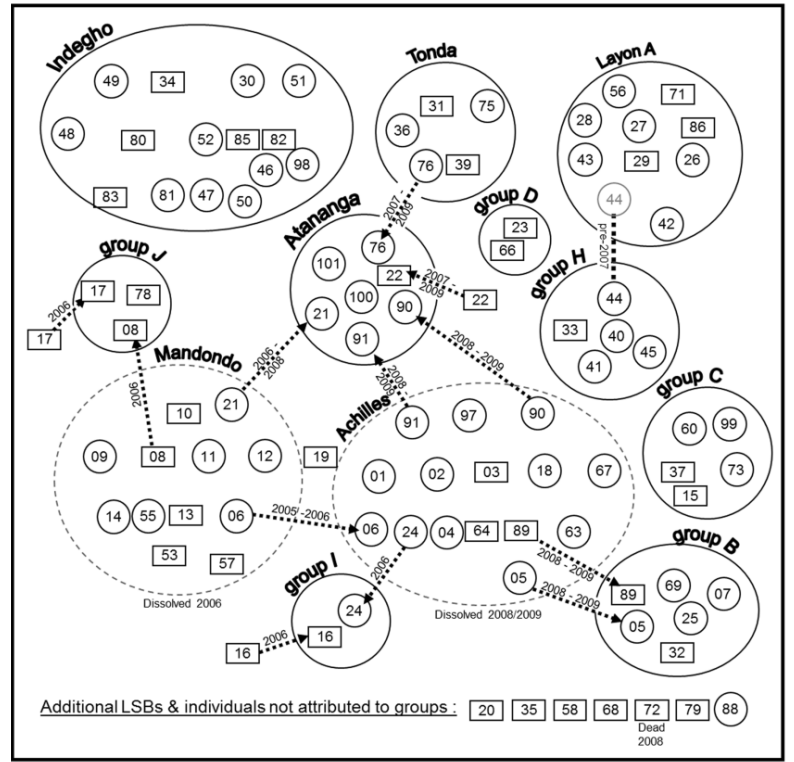

Female

$\square$ Male

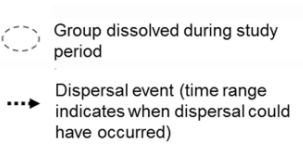
indicates when dispersal could
have occurred) (b)

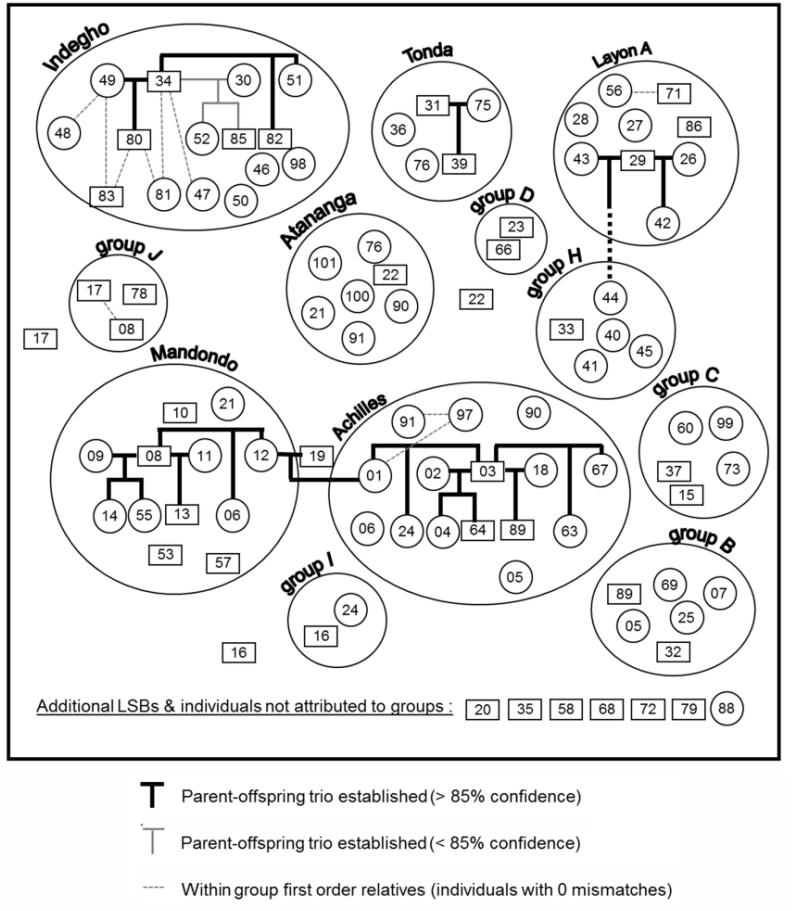

Figure 2. Individual gorillas are shown as circles (females) or rectangles (males). Social units are not displayed according to geographic proximity. When detected alone, we considered males G16, G17, G19, G20, G22, G35 and G72 as lone silverbacks. Males G58, G68 and G79 and female G88 could not be attributed to any social unit as we sampled them once and on their own (NB: for simplicity's sake, the "G" was removed from individual codes in the diagram). (a) Dispersal, dissolution and group formation events. Arrows indicate dispersal events; dotted lines encircling groups indicate group dissolutions. (b) Identified pedigrees for the various groups. Lines between gorillas indicate family relationships determined from genetic data.

nated from the dissolved Achilles group were not a motherdaughter pair. We also inferred eight cases of female group transfer and three cases of male transfer between social units and found one dead adult male (G72) (Fig. 2a).

Samples from 2 of the 12 groups contained only males, and so these are possible all-male non-breeding groups (Figs. 1, 2a). Five groups contained both males and females, but we did not identify any pedigree relationships between these individuals using our criteria, and in the absence of age data we cannot be sure whether these are breeding or non-breeding groups (Levréro et al., 2006). From the pedigree analysis, we identified 15 parent-offspring trios in the remaining five groups (Fig. 2b), so we can be confident that this is the minimum number of breeding groups existing over the course of the study period. Although not set as a prior, parent-offspring trio assignments always identified candidate parents and offspring within the same group, except in two cases: (1) we found that a lone silverback (G19) is the probable father of a breeding female (G01) found in the Achilles group (with female G12 from the Mandondo group as the assigned mother), and (2) we identified the LayonA group silverback (G29) along with LayonA female G43 as the probable parents of group H female G44 (Fig. 2b).

\subsection{Female relatedness}

In the first permutation analysis where average female relatedness was compared to the permuted relatedness values of all adults across the study area, we found that females were on average more related to each other $(R=0.017$, $\mathrm{SD} \pm 0.139, N=36)$ than expected by chance $(p=0.017)$. Second, we also found that average dyadic relatedness of females within groups $(R=0.043, \mathrm{SD} \pm 0.182, N=35)$ was significantly higher than when randomly assigning individuals to groups $(p=0.045)$. This relationship still held when restricting this analysis to only the well-sampled breeding groups (Achilles, Indegho, LayonA group and Tonda) during the same time period $(R=0.054, \mathrm{SD} \pm 0.192, p=0.024$, $N=24)$. Third, the average dyadic relatedness of females within groups was not significantly higher than when assigning individuals to groups while accounting for geographic distance using the Lynch and Ritland estimator $(p=0.161)$, but was when using the Queller and Goodnight estimator $(p=0.021)$.

Specific examples of potential kin associations between females can also be extracted from the data. In the Indegho and Achilles groups, we identified one and two cases, respectively, where pairs of females shared an allele at all 
loci and thus represent potential mother-offspring, but mismatched the breeding male at two loci or more (Fig. 2b). Thus, these three females are not daughters of the silverback but either immigrated into groups which contained their kin; transferred into groups with their daughters; or, following group dissolution due to the death of a silverback, joined the same new silverback. Furthermore, putative half-sisters G06 and G01 (sharing Mandondo female G12 as their mother) both apparently immigrated into the Achilles group. In the Achilles group we also identify females G91, G97 and G01 as first-order relatives (potential mother-daughters or sisters) who were also unrelated to the group silverback.

\section{Discussion}

\subsection{Group dynamics and dispersal}

By using genetic analysis to monitor multiple groups over several years, we indirectly observed the dynamics of group formation, group dissolution and individual movements in WLGs. As we have observed not only harem groups but also probable multi-male and mixed-sex non-reproductive groups at Loango, our results add to the growing body of evidence suggesting that the structure of WLG society contains various types of social units (Gatti et al., 2004; Levréro et al., 2006; Robbins et al., 2004). Furthermore, not all males in the reproductive groups are sons of the silverback, atypical for classical harem social units (Fig. 2b). Over a 5-year period, while genetically monitoring 18 social units, we inferred the dissolution of two groups, the formation of two groups, the death of one male and the dispersal of 13 individuals between social units. Taking into account the limits of our approximated number of recorded gorilla social unit years as detailed in the methods, the rate of group dissolution (2 in 19 social unit years: $11 \%$ ) is similar to that observed at Mbeli Bai (5 dissolutions in 63 gorilla group years: $8 \%$ ) (Robbins et al., 2004), as is the rate of female transfer: 8 cases in 19 social unit years $(42 \%)$ at Loango and 27 incidences in 63 group years $(43 \%)$ at Mbeli Bai (Stokes et al., 2003). It is important to note that these results represent the minimum number of dispersal events, social unit formations and dissolutions and that with increased sampling it is likely that more such events would be revealed. Similarly, this suggests that our rates of group dissolution and female transfer underestimate the true rates at Loango.

\subsection{Female relatedness}

In many species, there are clear benefits for females to reside with female kin (Silk, 2009), so much so that most mammals are female philopatric (Greenwood, 1980; Pusey and Packer, 1987). Despite low levels of feeding competition and no evidence of between-group competition for resources, related mountain gorilla females exhibit more affiliative behaviours towards kin than non-kin when they live in the same group
(Watts, 1994a) and female kin are more likely to form coalitions than non-kin in competition over food (Harcourt and Stewart, 1989; Watts, 2001). However, no studies have yet tested to see whether residing with and supporting kin improves their fitness (as shown in other species; meerkats: Clutton-Brock et al., 2010; white-faced capuchins: Fedigan et al., 2008; howler monkeys: Pope, 2000; savannah baboons: Van Horn et al., 2007).

As suggested in previous studies, our analyses imply that female WLGs do not disperse far from their natal range as the average relatedness of females across all groups at the study site was higher than expected by chance (Bradley et al., 2007; Douadi et al., 2007; Harcourt and Stewart, 2007; Jeffery et al., 2007). Furthermore, we found that, assuming that all groups at Loango are equally viable dispersal options, females co-occur in groups containing their kin more often than would be expected than if they dispersed randomly. When we attempted to correct for group proximity, we obtained mixed results depending on the relatedness estimator used. Therefore, we cannot distinguish between whether the patterns we observe are due to a process of related females dispersing to geographically proximate groups or whether there may be other factors that lead to related females occurring in the same post-dispersal groups. It is important to note, however, that due to limitations in the test procedure our geographic proximity permutation test is biased towards permuting co-residing females together. As we found that females within groups are more likely to be related than expected by chance, this means that our geographic proximity permutation test is biased towards finding related individuals occurring in the same group and therefore finding a non-significant result. This suggests that the significant result obtained with one of the two relatedness estimators may indeed be the more accurate of the two results and that females may not be basing their dispersal decisions on just geographic proximity. Female dispersal decisions are likely influenced by male quality (Breuer et al., 2010, 2012; Caillaud et al., 2008) and probably by habitat/vegetation composition familiarity as seen in mountain gorillas (Ganas et al., 2004; Guschanski et al., 2009). Our tests cannot determine the primary driver(s) of female dispersal, nor rule out that co-residency of female kin may be a secondary effect of other drivers of female dispersal decisions. Further long-term studies taking these factors into account will better elucidate how female relatives end up together post-dispersal.

Finding related post-dispersal females in the same group is consistent with a previous study on a smaller geographic scale (Bradley et al., 2007), but not with two others (Douadi et al., 2007; Inoue et al., 2013). The studies which failed to find a non-random distribution of female kin relied on assessment of dyadic kin relationships, an approach known to be less reliable than average group relatedness measures due to high levels of noise in the pairwise estimators (Csillery et al., 2006). Furthermore, as most samples came from a onetime sampling of unhabituated animals, some group mem- 
bers may have been omitted in the sampling, dampening the signal of within-group female relatedness. Finally, the different conclusions may be a result of smaller sample sizes in previous studies as it is well established that small sample sizes lead to reductions in statistical power. Inoue et al. (2013) included 12 adult females from 5 groups in their analyses and Douadi et al. (2007) included 14 females from 6 groups, while Bradley et al. (2007) analysed 22 adult females from 8 groups and we evaluated 35 adult females from 8 groups. We also inferred several instances of apparent natal and secondary transfer where females moved between groups either separately or together, which has also been observed in other populations (Robbins et al., 2004).

Bradley et al. (2007) suggest that post-dispersal female kin associations could occur in a scenario where female halfsiblings (daughters of their group's silverback) reach dispersal age at similar times and transfer into the same group. We have evidence here that, in at least one case, the cooccurrence of half-siblings G01 and G06 in Achilles group occurred over a longer time period as G01 had already produced at least one offspring with the Achilles silverback when G06 immigrated into the group. Thus, females may not need to be in the same age cohort (and possibly may have never even co-resided in their natal group) but may still transfer into the same group over a longer time period; further long-term monitoring is needed to determine the kin knowledge of females prior to dispersal.

It has also been suggested that related females may coreside in a new group if they co-transfer after the disintegration of their natal group (Bradley et al., 2007). In our data set we have one case of two Achilles group females (not a mother-daughter pair) who transferred to the Atananga group. We are unable, however, to specify the degree of relatedness of these females other than to say that they are not daughters of the Achilles silverback and thus that their transfer was probably a secondary dispersal event. Further sampling across the Loango study site to determine into which groups the Achilles females emigrated after the group dissolution should better elucidate the pattern of female kin associations in WLGs.

\section{Conclusions}

Our multi-year genetic monitoring study reinforces previous findings that WLGs exhibit dynamic group compositions and social structures as observed across their range. We also show that females, despite natal and secondary dispersal, appeared to retain associations with their same-sex kin post-emigration as females co-reside in groups containing female kin. The lack of long-term behavioural data on multiple western gorilla groups limits the inferences we can make on the function of these kin relations; however, dispersal need not be treated as an impediment to the possibility of kin-biased behaviours in the species.

\section{The Supplement related to this article is available online at doi:10.5194/pb-1-29-2014-supplement.}

Author contributions. M. Arandjelovic, L. Vigilant, M. M. Robbins and C. Boesch conceived and designed the study. J. Head, C. Boesch and M. M. Robbins collected samples, provided field information and field-based infrastructure. L. Vigilant contributed reagents and materials. M. Arandjelovic performed the experiments and analyses. M. Arandjelovic prepared the manuscript with contributions from all co-authors.

Acknowledgements. We thank the Agence Nationale des Parcs Nationaux (ANPN) and the Centre National de la Recherche Scientifique et Technique (CENAREST) of Gabon for permission to conduct our research in Loango National Park. We also thank L. Rabanal, L. Makaga, E. R. Guizard, E. Fairet, M. Gregoire, L. Rankin, E. Wright and the other field assistants of the Loango Ape project for their help collecting the samples. We are very grateful to D. Lukas, D. Caillaud, D. Morgan, C. Sanz, G. Schubert, O. Thalmann, B. J. Bradley, J. Ganas, K. Langergraber, T. Breuer, G. Campbell, J. Junker, A. Kalan, M. Whitten and C. Zacharias for helpful discussions, A. Abraham and C. Lang for laboratory assistance and R. Mundry and D. Lukas for statistical assistance and programming. We are also immensely grateful to the Editor and two anonymous reviewers for their constructive feedback and insightful suggestions which greatly improved this manuscript. This collaborative project is financially supported by, the Sociéte pour la Conservation et le Développement (SCD), Wildlife Conservation Society (WCS), and the Max Planck Society.

Edited by: J. Tung

Reviewed by: two anonymous referees

\section{References}

Arandjelovic, M., Guschanski, K., Schubert, G., Harris, T. R., Thalmann, O., Siedel, H., and Vigilant, L.: Two-step multiplex polymerase chain reaction improves the speed and accuracy of genotyping using DNA from noninvasive and museum samples, Mol. Ecol. Resour., 9, 28-36, 2009.

Arandjelovic, M., Head, J., Kuehl, H., Boesch, C., Robbins, M. M., Maisels, F., and Vigilant, L.: Effective non-invasive genetic monitoring of multiple wild western gorilla groups, Biol. Conserv., 143, 1780-1791, 2010.

Bermejo, M.: Home-range use and intergroup encounters in western gorillas (Gorilla g. gorilla) at Lossi Forest, North Congo, Am. J. Primatol., 64, 223-232, 2004.

Blouin, M. S.: DNA-based methods for pedigree reconstruction and kinship analysis in natural populations, Trends in Ecology and Evolution, 18, 503-511, 2003.

Blouin, M. S., Parsons, M., Lacaille, V., and Lotz, S.: Use of microsatellite loci to classify individuals by relatedness, Mol. Ecol., 5, 393-401, 1996. 
Boesch, C.: The real chimpanzee: sex strategies in the forest, Cambridge University Press, Cambridge, UK, 2009.

Bradley, B. J., Boesch, C., and Vigilant, L.: Identification and redesign of human microsatellite markers for genotyping wild chimpanzees (Pan troglodytes verus) and gorilla (Gorilla gorilla gorilla) DNA from feces, Conserv. Genet., 1, 289-292, 2000.

Bradley, B. J., Doran-Sheehy, D. M., Lukas, D., Boesch, C., and Vigilant, L.: Dispersed male networks in gorillas, Curr. Biol., 14, 510-513, 2004.

Bradley, B. J., Doran-Sheehy, D. M., and Vigilant, L.: Potential for female kin associations in wild western gorillas despite female dispersal, P. Roy. Soc. B Biol. Sci., 274, 2179-2185, 2007.

Breuer, T.: Winona's search for the right silverback: Insights about female strategies from a natural rain forest clearing in northern Congo, in: Among african apes: stories and photos from the field, edited by: Robbins, M. M. and Boesch, C., University of California Press, 2010.

Breuer, T., Robbins, A. M., Olejniczak, C., Parnell, R. J., Stokes, E. J., and Robbins, M. M.: Variance in the male reproductive success of western gorillas: acquiring females is just the beginning, Behav. Ecol. Sociobiol., 64, 515-528, 2010.

Breuer, T., Robbins, A. M., Boesch, C., and Robbins, M. M.: Phenotypic correlates of male reproductive success in western gorillas, J. Hum. Evol., 62, 466-472, 2012.

Caillaud, D., Levréro, F., Gatti, S., Ménard, N., and Raymond, M.: Influence of male morphology on male mating status and behavior during interunit encounters in western lowland gorillas, Am. J. Phys. Anthropol., 135, 379-388, 2008.

Chapais, B.: Primate nepotism: what is the explanatory value of kin selection?, Int. J. Primatol., 22, 203-229, 2001.

Cipolletta, C.: Effects of group dynamics and diet on the ranging patterns of a western gorilla group (Gorilla gorilla gorilla) at Bai Hokou, Central African Republic, Am. J. Primatol., 64, 193-205, 2004.

Clutton-Brock, T. and Huchard, E.: Social competition and its consequences in female mammals, J. Zool., 289, 151-171, 2013.

Clutton-Brock, T. H. and Lukas, D.: The evolution of social philopatry and dispersal in female mammals, Mol. Ecol., 21, 472-492, 2012.

Clutton-Brock, T. H., Hodge, S. J., Flower, T. P., Spong, G. F., and Young, A. J.: Adaptive suppression of subordinate reproduction in cooperative mammals, The American naturalist, 176, 664$673,2010$.

Csillery, K., Johnson, T., Beraldi, D., Clutton-Brock, T., Coltman, D., Hansson, B., Spong, G., and Pemberton, J. M.: Performance of marker-based relatedness estimators in natural populations of outbred vertebrates, Genetics, 173, 2091-2101, 2006.

Doran-Sheehy, D. M., Greer, D., Mongo, P., and Schwindt, D.: Impact of ecological and social factors on ranging in western gorillas, Am. J. Primatol., 64, 207-222, 2004.

Douadi, M. I., Gatti, S., Levrero, F., Duhamel, G., Bermejo, M., Vallet, D., Menard, N., and Petit, E. J.: Sex-biased dispersal in western lowland gorillas (Gorilla gorilla gorilla), Mol. Ecol., 16, 2247-2259, 2007.

Fedigan, L. M., Carnegie, S. D., and Jack, K. M.: Predictors of reproductive success in female white-faced capuchins (Cebus capucinus), Am. J. Phys. Anthropol., 137, 82-90, 2008.

Ganas, J., Robbins, M. M., Nkurunungi, J. B., Kaplin, B. A., and McNeilage, A.: Dietary variability of mountain gorillas in
Bwindi Impenetrable National Park, Uganda, Int. J. Primatol., 25, 1043-1072, 2004.

Gatti, S., Levréro, F., Ménard, N., and Gautier-Hion, A.: Population and group structure of western lowland gorillas (Gorilla gorilla gorilla) at Lokoué, Republic of Congo, Am. J. Primatol., 63, 111-123, 2004.

Greenwood, P. J.: Mating systems, philopatry, and dispersal in birds and mammals, Anim. Behav., 28, 1140-1162, 1980.

Guschanski, K., Caillaud, D., Robbins, M. M., and Vigilant, L.: Females shape the genetic structure of a gorilla population, Curr. Biol., 18, 1809-1814, 2008.

Guschanski, K., Vigilant, L., McNeilage, A., Gray, M., Kagoda, E., and Robbins, M. M.: Counting elusive animals: Comparing field and genetic census of the entire mountain gorilla population of Bwindi Impenetrable National Park, Uganda, Biol. Conserv., 142, 290-300, 2009.

Handley, L. J. L. and Perrin, N.: Advances in our understanding of mammalian sex-biased dispersal, Mol. Ecol., 16, 1559-1578, 2007.

Harcourt, A. H. and Stewart, K. J.: Functions of alliances in contests within wild gorilla groups, Behaviour, 109, 176-190, 1989.

Harcourt, A. H. and Stewart, K. J.: Gorilla society: conflict, compromise, and cooperation between the sexes, University of Chicago Press, Chicago, 2007.

Hatchwell, B. J.: Cryptic kin selection: kin structure in vertebrate populations and opportunities for kin-directed cooperation, Ethology, 116, 203-216, 2010.

Head, J., Boesch, C., Makaga, L., and Robbins, M. M.: Sympatric Chimpanzees (Pan troglodytes troglodytes) and Gorillas (Gorilla gorilla gorilla) in Loango National Park, Gabon: Dietary Composition, Seasonality, and Intersite Comparisons, Int. J. Primatol., 32, 755-775, 2011.

Head, J., Boesch, C., Robbins, M. M., Rabanal, L. I., Makaga, L., and Kuehl, H.: Effective socio-demographic population assessment of elusive species in ecology and conservation management, Ecol. Evol., 3, 2903-2916, 2013.

Inoue, E., Akomo-Okoue, E. F., Ando, C., Iwata, Y., Judai, M., Fujita, S., Hongo, S., Nze-Nkogue, C., Inoue-Murayama, M., and Yamagiwa, J.: Male genetic structure and paternity in western lowland gorillas (Gorilla gorilla gorilla), Am. J. Phys. Anthropol., 151, 583-588, 2013.

Jeffery, K. J., Abernethy, K. A., Tutin, C. E., Anthony, N. A., and Bruford, M. W.: Who killed Porthos? Genetic tracking of a gorilla death, Integrative Zoology, 2, 111-119, 2007.

Koenig, A. and Borries, C.: Hominoid dispersal patterns and human evolution, Evol. Anthropol., 21, 108-112, 2012.

Konovalov, D. A., Manning, C., and Henshaw, M. T.: KINGROUP: a program for pedigree relationship reconstruction and kin group assignments using genetic markers, Mol. Ecol. Notes, 4, 779782, 2004.

Langergraber, K., Mitani, J., and Vigilant, L.: Kinship and social bonds in female chimpanzees (Pan troglodytes), Am. J. Primatol., 71, 840-851, 2009.

Langergraber, K. E., Mitani, J. C., and Vigilant, L.: The limited impact of kinship on cooperation in wild chimpanzees, P. Natl. Acad. Sci. USA, 104, 7786-7790, 2007.

Levréro, F., Gatti, S., Ménard, N., Petit, E., Caillaud, D., and Gautier-Hion, A.: Living in nonbreeding groups: An alternative 
strategy for maturing gorillas, Am. J. Primatol., 68, 275-291, 2006.

Lukas, D., Reynolds, V., Boesch, C., and Vigilant, L.: To what extent does living in a group mean living with kin?, Mol. Ecol., 14, 2181-2196, 2005.

Lynch, M. and Ritland, K.: Estimation of pairwise relatedness with molecular markers, Genetics, 152, 1753-1766, 1999.

Magliocca, F., Querouil, S., and Gautier-Hion, A.: Population structure and group composition of western lowland gorillas in NorthWestern Republic of Congo, Am. J. Primatol., 48, 1-14, 1999.

Manly, B.: Randomization, bootstrap and monte carlo methods in biology, Chapman \& Hall, London, 1997.

Morin, P. A., Chambers, K. E., Boesch, C., and Vigilant, L.: Quantitative PCR analysis of DNA from noninvasive samples for accurate microsatellite genotyping of wild chimpanzees (Pan troglodytes verus), Mol. Ecol., 10, 1835-1844, 2001.

Nsubuga, A. M., Robbins, M. M., Roeder, A. D., Morin, P. A., Boesch, C., and Vigilant, L.: Factors affecting the amount of genomic DNA extracted from ape faeces and the identification of an improved sample storage method, Mol. Ecol., 13, 2089-2094, 2004.

Parnell, R. J.: Group size and structure in western lowland gorillas (Gorilla gorilla gorilla) at Mbeli Bai, Republic of Congo, Am. J. Primatol., 56, 193-206, 2002.

Pope, T. R.: Reproductive success increases with degree of kinship in cooperative coalitions of female red howler monkeys (Alouatta seniculus), Behav. Ecol. Sociobiol., 48, 253-267, 2000.

Pritchard, J. K., Stephens, M., and Donnelly, P.: Inference of population structure using multilocus genotype data, Genetics, 155 , 945-959, 2000.

Pusey, A. E. and Packer, C.: Dispersal and philopatry, in: Primate Societies, edited by: Smuts, B. B., Cheney, D. L., Seyfarth, R. M., Wrangham, R. W., and Struhsaker, T. T., Chicago University Press, Chicago, 250-266, 1987.

Queller, D. C. and Goodnight, K. F.: Estimating relatedness using genetic markers, Evolution, 43, 258-275, 1989.

R Core Team: R: A language and environment for statistical computing, Vienna, Austria, 2013.

Robbins, A. M., Gray, M., Basabose, A., Uwingeli, P., Mburanumwe, I., Kagoda, E., and Robbins, M. M.: Impact of male infanticide on the social structure of mountain gorillas, PLoS ONE, 8, e78256, doi:10.1371/journal.pone.0078256, 2013.

Robbins, M. M.: Gorillas: diversity in ecology and behavior, in: Primates in Perspective, 2nd Edn., edited by: Campbell, C., Fuentes, A., MacKinnon, K., Bearder, S. K., and Stumpf, R. M., Oxford University Press, New York, 2010.

Robbins, M. M., Bermejo, M., Cipolletta, C., Magliocca, F., Parnell, R. J., and Stokes, E. J.: Social structure and life-history patterns in western gorillas (Gorilla gorilla gorilla), Am. J. Primatol., 64, 145-159, 2004.

Rubenstein, D. I.: Ecology and sociality in horses and zebras, in: Ecological Aspects of Social Evolution, edited by: Rubenstein, D. I. and Wrangham, R. W., Princeton University Press, Princeton, New Jersey, 1986.

Schaller, G. B.: The mountain gorilla: ecology and behaviour, University of Chicago, Chicago, 1963.
Silk, J. B.: Nepotistic cooperation in non-human primate groups, Philos. T. R. Soc. B, 364, 3243-3254, 2009.

Silk, J. B., Altmann, J., and Alberts, S. C.: Social relationships among adult female baboons (Papio cynocephalus) I. Variation in the strength of social bonds, Behav. Ecol. Sociobiol., 61, 183195, 2006.

Stokes, E. J., Parnell, R. J., and Olejniczak, C.: Female dispersal and reproductive success in wild western lowland gorillas (Gorilla gorilla gorilla), Behav. Ecol. Sociobiol., 54, 329-339, 2003.

Swedell, L. and Tesfaye, T.: Infant mortality after takeovers in wild Ethiopian hamadryas baboons, Am. J. Primatol., 60, 113-118, 2003.

Thierry, B.: Primate socioecology, the lost dream of ecological determinism, Evol. Anthropol., 17, 93-96, 2008.

Tutin, C. E. G.: Ranging and social structure of lowland gorillas in the Lope Reserve, Gabon, in: Great ape societies, edited by: McGrew, W. C., Marchant, L. F., and Nishida, T., Cambridge University Press, Cambridge, 58-70, 1996.

Van Horn, R. C., Buchan, J. C., Altmann, J., and Alberts, S. C.: Divided destinies: group choice by female savannah baboons during social group fission, Behav. Ecol. Sociobiol., 61, 1823-1837, 2007.

Van Horn, R. C., Altmann, J., and Alberts, S. C.: Can't get there from here: inferring kinship from pairwise genetic relatedness, Anim. Behav., 75, 1173-1180, 2008.

Voigt, C. C. and Streich, W. J.: Queuing for harem access in colonies of the greater sac-winged bat, Anim. Behav., 65, 149-156, 2003.

Waits, L. P., Luikart, G., and Taberlet, P.: Estimating the probability of identity among genotypes in natural populations: cautions and guidelines, Mol. Ecol., 10, 249-256, 2001.

Walsh, P. D., Tutin, C. E. G., Baillie, J. E. M., Maisels, F., Stokes, E. J., and Gatti, S.: Gorilla gorilla ssp. gorilla The IUCN Red List of Threatened Species, Version 2014.2, available at: www. iucnredlist.org (last access: 6 September 2014), 2008.

Watts, D. P.: Agonistic relationships between female mountain gorillas (Gorilla gorilla berengei), Behav. Ecol. Sociobiol., 34, 347-358, 1994a.

Watts, D. P.: Social relationships of immigrant and resident female mountain gorillas, II: Relatedness, residence, and relationships between females, Am. J. Primatol., 32, 13-30, 1994b.

Watts, D. P.: Causes and consequences of variation in male mountain gorilla life histories and group membership, in: Primate males: causes and consequences of variation in group composition, edited by: Kappeler, P. M., Cambridge University Press, Cambridge, 169-179, 2000.

Watts, D. P.: Social relationships of female mountain gorillas, in: Mountain gorillas: Three decades of research at Karisoke, edited by: Robbins, M. M., Sicotte, P., and Stewart, K. J., Cambridge University Press, Cambridge, 215-240, 2001.

Wrangham, R.: On the evolution of ape social systems, Soc. Sc. Inform., 18, 336-368, 1979. 